\title{
Associação entre Fumar e Progressão para Complicações Respiratórias Graves em Pacientes com Covid-19
}

https://doi.org/10.32635/2176-9745.RBC.2020v66n2.974

Association between Smoking and Progression to Severe Disease in COVID-19 Patients
Asociación entre Tabaquismo y Progresión a Enfermedad Grave en Pacientes con Covid-19

André Salem Szklo ${ }^{1}$

\section{INTRODUÇÃO}

O tabagismo é considerado um grave problema de saúde pública ${ }^{1}$ e uma das suas complicações é a redução da capacidade respiratória do indivíduo. Além disso, fumar aumenta o risco de infecçóes bacterianas e virais, tais como doença pulmonar pneumocócica invasiva, influenza e tuberculose. O tabagismo é, ainda, o principal fator de risco associado ao desenvolvimento da doença pulmonar obstrutiva crônica (DPOC), sendo responsável, no Brasil, por $88 \%$ das mortes por DPOC entre os homens e $81 \%$ das mortes entre as mulheres ${ }^{2}$. O comportamento de fumar é associado também a outras doenças crônicas não transmissíveis (DCNT), como cardiopatias, diabetes, hipertensão arterial e câncer ${ }^{1}$.

Essas comorbidades representam importantes fatores de risco para as complicaçôes e óbito pelo coronavírus 2019 (Covid-19). Este texto busca fazer um resumo crítico dos dados disponíveis e publicados em revistas indexadas sobre a associação entre fumar e evolução de complicaçóes respiratórias relacionadas ao Covid-19.

\section{DESENVOLVIMENTO}

Todos os estudos publicados até o momento (3/4/2020) vêm da China, onde a epidemia começou primeiro.

Uma revisão da literatura realizada em meados de março de 2020 (17/3/2020) encontrou cinco artigos relevantes ${ }^{3}$. Três dos cinco trabalhos não mostraram diferenças estatisticamente significativas entre fumantes e não fumantes em termos de progressão da doençç ${ }^{4-6}$ e um estudo, infelizmente, náo relatou o valor de p encontrado para a comparação estatística entre fumantes e não fumantes na progressão da doença ${ }^{7}$. Finalmente, um estudo que analisou 78 pacientes diagnosticados com pneumonia associada ao Covid-19 e que estavam no hospital por duas semanas verificou que a chance de progressão negativa da doença (inclusive até a morte) era cerca de 14 vezes maior entre as indivíduos com histórico de tabagismo em comparação com aqueles que não fumavam ${ }^{8}$. Esse foi o fator de risco mais forte entre os todos os fatores de progressão analisados nesse estudo.

Vale a pena assinalar que, apesar de um estudo náo ter mostrado a comparação estatística e três estudos publicados terem encontrado resultados náo conclusivos do ponto de vista estatístico, os seus achados sugerem piores prognósticos entre os fumantes ( $v$ s não fumantes). Por exemplo, o estudo que avaliou 1.099 pacientes e que não relatou o valor de $\mathrm{p}$ da comparação estatística constatou que os fumantes tiveram aproximadamente o triplo de chance de evoluírem para um prognóstico pior (incluindo a morte) quando comparados aos não fumantes ${ }^{7}$. Um outro estudo que avaliou as variáveis prognósticas de morte entre 191 pessoas hospitalizadas com Covid-19 e que não encontrou associação estatística com o tabagismo mostrou que os fumantes apresentaram mais do que o dobro de chance de morrer quando comparados aos não fumantes ${ }^{6}$.

Em resumo, apesar do pequeno número de estudos disponíveis até o momento, a revisão sistemática da literatura ${ }^{3}$ apontou para uma provável associação entre tabagismo e progressáo negativa de complicaçóes respiratórias relacionadas ao Covid-19.

Uma metanálise 9 realizada com artigos publicados até o dia de 9 de março de 2020 e publicada no mesmo período da revisão sistemática mencionada anteriormente ${ }^{3}$ incluiu um artigo que não constava dessa revisão ${ }^{10}$. Nesse artigo, entre 53 pacientes analisados, havia apenas dois fumantes e ambos tiveram uma evoluçáo melhor da doença. Por outro lado, na revisão sistemática ${ }^{3}$ aparece um

' Tecnologista da Divisão de Pesquisa Populacional do Instituto Nacional de Câncer José Alencar Gomes da Silva (INCA). Orcid iD: https//orcid.org/0000-00031903-6188

Endereço para correspondência: André Salem Szklo. Rua Marquês de Pombal, 125 - 70 andar - Centro. Rio de Janeiro (RJ), Brasil. CEP 20230-240. E-mail: aszklo@inca.gov.br 
outro artigo publicado em 12 de março e que apresenta um cenário mais desfavorável para 11 fumantes de um total de 191 pacientes analisados ${ }^{6}$.

$\mathrm{O}$ resultado da metanálise não achou uma relação estatisticamente significativa entre fumo ativo e severidade do Covid-19. No entanto, o estudo mais robusto com mais de mil pacientes, incluído nessa metanálise, encontrou uma razão de chances (odds ratio - OR) para severidade do Covid-19 entre fumantes e não fumantes de 1,51 $(0,97-2,36)^{7}$. Ou seja, o limite inferior da medida de associação está bem próximo do valor unitário e, além disso, o conjunto de valores mais prováveis baseados na curva da distribuição aponta claramente para um cenário pior para os fumantes.

Mais recentemente, surgiu um outro artigo publicado no dia 26 de março ${ }^{11}$. Na verdade, trata-se do mesmo estudo citado anteriormente 7 : "o que avaliou 1.099 pacientes e que concluiu que os fumantes tiveram aproximadamente o triplo de chance de evoluírem para um prognóstico pior (incluindo a morte) quando comparados aos náo fumantes", com os seguintes complementos: 1) 1.599 pacientes hospitalizados vs 1.099 pacientes hospitalizados; 2) pacientes provenientes de 575 hospitais em 31 províncias da China vs pacientes provenientes de 552 hospitais em 30 províncias; 3 ) o artigo mais recente se preocupou primordialmente em analisar a relação entre a presença de comorbidades e a evoluçáo para um desfecho pior, e constatou, por exemplo, o efeito independente da presença de DPOC, hipertensão, diabetes ou câncer sobre a piora do prognóstico (necessidade de ventilação mecânica, Unidade de Tratamento Intensivo - UTI ou morte). Esse estudo avaliou, ainda, o efeito negativo independente de o indivíduo apresentar pelo menos uma comorbidade (ou duas ou mais comorbidades) sobre a evolução do seu estado de saúde. Além da análise específica por comorbidades já citadas, tem-se informação sobre doenças cardiovasculares, doenças renais, doenças autoimunes e hepatite; 4) o mais interessante e novo foi que os autores ajustaram todas as análises pela idade e pelo status de fumante do paciente. Ou seja, apesar de os autores terem tratado o fato de ser fumante ou náo apenas como variável de confundimento da análise principal, eles obtiveram também o efeito independente de o paciente ser ou não fumante (i.e., sem considerar as diferenças de idade e a presença ou náo de comorbidades entre fumantes e não fumantes) sobre a evolução para um desfecho mais negativo (necessidade de ventilação mecânica, UTI ou morte). Em outras palavras, consegue-se entender se o fumante vai evoluir pior não apenas porque, como se sabe, ele tem uma frequência maior de comorbidades do que o náo fumante. Os resultados obtidos apontam que, levando-se em consideração os diferentes tempos de seguimento dos pacientes, independentemente da idade, de ter ou não DPOC, diabetes, hipertensão ou câncer, os fumantes na vida têm em média um risco $67 \%$ maior de evoluir para um desfecho pior do que os não fumantes (hazard ratio - HR 1,67 (1,01-2,76)). Além disso, independentemente de ter alguma comorbidade (uma, duas ou mais comorbidades) ou não, novamente o fumante tem em média um risco $73 \%$ maior de prognóstico ruim quando comparado aos náo fumantes (hazard ratio - HR 1,73 (1,05-2,87)).

A maioria dos estudos publicados até agora tem encontrado um maior percentual de homens entre os pacientes com Covid-19, o que poderia sugerir também que, como os homens fumam bem mais do que as mulheres na China, o fumo também estaria associado com uma maior incidência da doença entre os fumantes ${ }^{12}$.

No entanto, a proporção de fumantes entre os doentes é muito menor do que a esperada para a população geral chinesa. Em estudo recente, por exemplo, cerca de 7\% eram fumantes/ex-fumantes, quando a proporção esperada de fumantes deveria ter sido de cerca de 30\% (50\% entre homens e $2 \%$ entre mulheres ${ }^{13}$, se fumantes tivessem tido a mesma probabilidade de se infectarem do que os não fumantes ${ }^{11}$. Em outras palavras, pelos dados apresentados/ coletados, muitos homens que chegaram com Covid-19 nos hospitais não eram fumantes.

$\mathrm{O}$ que pode estar ocorrendo para que aparentemente os fumantes não se infectem? Não temos, no momento, a resposta, uma vez que grande parte dos fatores prognósticos de evolução da doença poderia estar mais ou menos relacionada também com aqueles associados à predisposição para se infectarem.

Será que nem todos os fumantes com Covid-19 estão sendo identificados? Por exemplo, pode ser que os fumantes, geralmente muito mais desprovidos do ponto de vista socioeconômico, não cheguem nem mesmo a serem internados antes de adoecer e/ou vir a falecer, não constando dessa forma dos pacientes hospitalizados selecionados para os estudos posteriormente publicados ("viés de sobrevida").

Ou será que os pacientes com Covid-19 não estão sendo classificados corretamente quanto ao seu "estado de fumante" e isso está ocorrendo com mais frequência entre fumantes do que não fumantes (erro de classificação diferencial da exposição)?

Ou seria uma explicação mais comportamental, tal como, por exemplo, os fumantes estarem mais atentos para não se exporem ao vírus por saberem que terão um pior prognóstico de evolução da doença? Ou ainda os fumantes estarem em menor frequência em ambientes mais confinados e com grande número de pessoas por terem que fumar no espaço aberto (segundo a Lei de Ambientes 
Livres na China - "Smoking is completely prohibited in at least 28 indoor public places, including medical facilities, restaurants, bars, and most public transportation. Designated smoking areas are permitted in other specified places, such as long-distance transport. Sub-national jurisdictions have the authority to implement local smoke free policies") ${ }^{14}$ ?

Ainda com relação ao tema da proporção de fumantes entre os pacientes infectados, saiu publicado no dia 24/3/2020 um artigo que se preocupou justamente em realizar uma metanálise com estudos publicados até 15 de fevereiro (ou seja, não utilizou os estudos mais recentes) para estimar a prevalência de doenças (incluindo o tabagismo) entre os pacientes com Covid-19 ${ }^{15}$. Obviamente, havia muita heterogeneidade entre os seis estudos incluídos ${ }^{4-5,7,11,15}$, e o resultado encontrado foi de uma prevalência combinada de tabagismo de $7,63 \%(3,83-12,43)$.

Os resultados do artigo de metanálise publicado em $24 / 3 / 2020^{15}$ reforçam a necessidade de se continuar estudando o perfil de pacientes com Covid-19 em outros países para entender até que ponto a incidência dessa doença estaria relacionada náo somente aos efeitos nocivos dos compostos presentes nos produtos derivados do tabaco (artigo publicado em 20 de março que apresenta a justificativa biológica dessa associação, uma vez que tabagistas apresentam uma maior expressão da enzima ACE2, receptor conhecido como sendo associado ao Covid-19) ${ }^{16}$, mas também a questóes comportamentais do fumante em face de diferentes realidades culturais e/ou diferentes cenários legislativos de combate ao tabagismo. Para viabilizar essa avaliação, seria fundamental a inclusão do fator de risco/doença "tabagismo" no rol das comorbidades apresentadas na Ficha de Notificação Nacional do Covid-19.

\section{CONCLUSÃO}

Finalmente, vale a pena reforçar que, desde a década de 1980, o Brasil desenvolve inúmeras açôes estruturadas para controle do tabagismo. No entanto, a epidemia do Covid-19 e os seus diversos impactos sobre o indivíduo, tais como o aumento de estresse em razão da incerteza quanto ao futuro (inclusive em função da recessão econômica) e o isolamento social em casa, representam um desafio potencial para a implementação efetiva das ações voltadas para a prevenção da iniciação, prevenção da recaída entre os milhóes de ex-fumantes brasileiros e estímulo à cessação ${ }^{17}$.

\section{CONTRIBUIÇÃO}

O autor contribuiu substancialmente em todas as etapas do manuscrito e aprovou a versão final a ser publicada.

\section{DECLARAÇÃO DE CONFLITO DE INTERESSES}

Nada a declarar.

\section{FONTES DE FINANCIAMENTO}

Não há.

\section{REFERÊNCIAS}

1. National Center for Chronic Disease Prevention and Health Promotion (US) Office on Smoking and Health. The health consequences of smoking - 50 years of progress: a report of the surgeon general. Atlanta (GA): Centers for Disease Control and Prevention (US); 2014.

2. Szklo AS, Iglesias RM, de Souza MC, et al. Understanding the relationship between sales of legal cigarettes and deaths: a case-study in Brazil. Prev Med. 2017 Jan;94:5559. doi: https://doi.org/10.1016/j.ypmed.2016.11.008

3. Vardavas CI, Nikitara K. COVID-19 and smoking: a systematic review of the evidence. Tob Induc Dis. 2020 Mar 20;18:20. doi: https://doi.org/10.18332/tid/119324

4. Zhang JJ, Dong X, Cao YY, et al. Clinical characteristics of 140 patients infected with SARS-CoV-2 in Wuhan, China. Allergy. 2020 Feb 19. doi: https://doi. org/10.1111/all.14238

5. Huang C, Wang Y, Li X, et al. Clinical features of patients infected with 2019 novel coronavirus in Wuhan, China. Lancet. 2020 Feb 15;395(10223):497-506. doi: https:// doi.org/10.1016/S0140-6736(20)30183-5

6. Zhou F, Yu T, Du R, et al. Clinical course and risk factors for mortality of adult inpatients with COVID-19 in Wuhan, China: a retrospective cohort study. Lancet. 2020 March 28;395(10229):1054-62. doi: https://doi. org/10.1016/S0140-6736(20)30566-3

7. Guan WJ, Ni ZY, Hu Y, et al. Clinical characteristics of coronavirus disease 2019 in China. N Engl J Med. 2020 Feb 28. doi: https://doi.org/10.1056/NEJMoa2002032

8. Liu W, Tao ZW, Lei W, et al. Analysis of factors associated with disease outcomes in hospitalized patients with 2019 novel coronavirus disease. Chin Med J (Engl). 2020 Feb 28. doi: https://doi.org/10.1097/ CM9.0000000000000775

9. Lippi G, Henry BM. Active smoking is not associated with severity of coronavirus disease 2019 (COVID-19). Eur J Intern Med. 2020 Mar 17. doi: https://doi. org/10.1016/j.ejim.2020.03.014

10. Yang $X, Y u ~ Y, X u ~ J$, et al. Clinical course and outcomes of critically ill patients with SARS-CoV-2 pneumonia in wuhan, China: a single-centered, retrospective, observational study. Lancet Respir Med. 2020 Feb 24. doi: https://doi.org/10.1016/ S2213-2600(20)30079-5 
11. Guan WJ, Liang WH, Zhao Y, et al. Comorbidity and its impact on 1590 patients with Covid-19 in China: a nationwide analysis. Eur Respir J. 2020 Mar 26:2000547. doi: https://doi.org/10.1183/13993003.00547-2020.

12. Cai H. Sex difference and smoking predisposition in patients with COVID-19. Lancet Respir Med. 2020 March 11;8(4):e20. doi: https://doi.org/10.1016/S22132600(20)30117-X. Erratum in: Lancet Respir Med. 2020 Apr;8(4):e26.

13. World Health Organization. WHO global report on trends in prevalence of tobacco use 2000-2025. 3rd. Geneva: WHO; 2019.

14. Tobacco Control Laws [Internet]. Washington, DC: Campaign for Tobacco-Free Kids; [date unknown]. Legislation by country: China; [update 2019 Nov 6; cited 2020 Apr 8]. Available from: https://www. tobaccocontrollaws.org/legislation/country/china/ summary

15. Emami A, Javanmardi F, Pirbonyeh N, et al. Prevalence of underlying diseases in hospitalized patients with COVID-19: a systematic review and meta-analysis. Arch Acad Emerg Med. 2020 March 24;8(1):e35.

16. Brake SJ, Barnsley K, Lu W, et al. Smoking upregulates angiotensin-converting enzyme-2 receptor: a potential adhesion site for novel coronavirus SARS-CoV-2 (Covid-19). J Clin Med. 2020 March 20;9(3):E841. doi: https://doi.org/10.3390/jcm9030841

17. Patwardhan P. COVID-19: risk of increase in smoking rates among England's 6 million smokers and relapse among England's 11 million ex-smokers. BJGP Open. 2020 Apr 7.pii:bjgpopen20X101067. doi: https://doi. org/10.3399/bjgpopen20X101067 